\title{
Research Paper: Preconditioning Effect of High-Intensity Aerobic Training on Myocardial Ischemia-Reperfusion Injury and Beclin-1 Gene Expression in Rats
}

\author{
Reza Ghahremani1 $^{1,2}$ (D), Iraj Salehi ${ }^{1}$ (D), Alireza Komaki ${ }^{1}$ (D), Arsalan Damirchi ${ }^{* *}$ (D) \\ 1. Neurophysiology Research Center, Hamadan University of Medical Sciences, Hamadan, Iran. \\ 2. Department of Exercise Physiology, Faculty of Sport Sciences, University of Guilan, Rasht, Iran.
}

\begin{tabular}{|c|c|}
\hline $\begin{array}{l}\text { Use your device to scan } \\
\text { and read the article online }\end{array}$ & ditration Ghahremani R, Salehi I, Komaki A, Damirchi A. Preconditioning Effect of High-Intensity Aerobic Training on \\
\hline 口ipipa & $\begin{array}{l}\text { Myocardial Ischemia-Reperfusion Injury and Beclin-1 Gene Expression in Rats. Physical Treatments. 2018; 8(2):115-121. } \\
\text { http://dx.doi.org/10.32598/ptj.8.2.115 }\end{array}$ \\
\hline 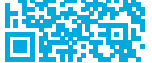 & dol' http://dx.doi.org/10.32598/ptj.8.2.115 \\
\hline
\end{tabular}

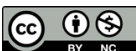

Article info:

Received: 22 Dec 2017

Accepted: 10 May 2018

Available Online: 01 Jul 2018

Keywords:

Aerobic training,

Cellular autophagy,

Ischemia-Reperfusion

injury, Cardioprotection

\section{A B S T RA C T}

Purpose: Ischemia-Reperfusion (IR) injury is one of the most common cardiac disorders leading to irreversible heart damage. Many underlying mechanisms seem to be involved, among which disruption of cellular autophagy balance. Since physical training has a beneficial effect on the improvement of autophagy balance, it may have a cardioprotective effect against IR injury. This study investigates the protective role of aerobic training from cardiac IR injury and the autophagy process as a possible mechanism.

Methods: Thirty-two male Wistar rats ( 8 weeks old) were divided into control, sham, control plus IR, and training plus IR groups ( 8 rats each). The training group was exercised aerobically on a treadmill for 8 weeks $(5 \mathrm{~d} / \mathrm{wk})$. After 8 weeks, the anesthetized rats underwent left thoracotomy (sham, control plus IR, and training plus IR groups) to access the left anterior descending coronary artery, which was occluded by a silk suture for $30 \mathrm{~min}$ and then released for $90 \mathrm{~min}$ of reperfusion (IR groups). Triphenyltetrazolium chloride staining was used to determine the infarct size. The gene expression of Beclin-1 was evaluated by real-time polymerase chain reaction. One-way ANOVA was used for statistical analysis with the significance level set at $\mathrm{P} \leq 0.05$.

Results: The cardiac infarct size was smaller in training plus IR (20.24 $\pm 5.7 \%)$ group compared to that in the control plus IR $(35.9 \pm 2.3 \%)$ group $(\mathrm{P} \leq 0.05)$. On the other hand, IR operation significantly increased the gene expression of Beclin-1, while exercise training prevented expression of the mentioned gene in training plus $I R$ group $(\mathrm{P} \leq 0.05)$

Conclusion: Aerobic training can protect the heart against Ischemia-Reperfusion injury. It seems that improvement of autophagy balance during IR injury may be involved in exercise-induced cardioprotection against Ischemia-Reperfusion.

\footnotetext{
* Corresponding Author:

Arsalan Damirchi, $P h D$.

Address: Department of Exercise Physiology, Faculty of Sport Sciences, University of Guilan, Rasht, Iran

Phone: +98 (13) 33690685

E-mail:damirchi@guilan.ac.ir
} 


\section{Highlights}

- Aerobic training can significantly increase cardiac muscle growth.

- An eight-week exercise training can protect the heart from Ischemia-Reperfusion injury.

- Improvement of autophagy balance may be involved in exercise-induced cardioprotection.

\section{Plain Language Summary}

Cardiovascular disease is one of the main causes of mortality around the world. These diseases endanger the lives of many people every year and cause huge costs for individuals and communities. One of these diseases is the transient stroke caused by various factors and has irreversible consequences. For this reason, providing a strategy that can reduce the risk of heart attacks can be useful. In summary, the present study indicates that regular exercise training causes cardiac muscle growth. Also, these exercises can reduce the injury caused by a temporary stroke and protect the cardiac tissue. Furthermore, our findings suggested that preserving the balance of the autophagy process during stroke seems to play a role in exercise-induced cardiac protection from this injury.

\section{Introduction}

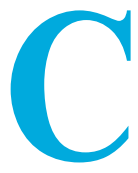

ardiovascular Diseases (CVDs) are among the main causes of worldwide deaths [13]. These diseases annually impose huge costs on individuals and communities. Therefore, the strategies to prevent CVDs are important for physicians and healthcare providers. The Ischemia-Reperfusion (IR) injury as a pathological condition associated with coronary artery disease is among the most frequent cause of heart failure that can generate irreversible complications [3]. Human epidemiological studies indicate that regular exercise provides protection from CVDs [4]. In addition, research revealed that exercise, as a preconditioning factor can protect the heart from IR injury [3, 5-7].

Increased glycolytic flux, improved nitric oxide signaling, the enhanced function of sarcolemmal or mitochondrial ATP-sensitive potassium channels, increased cytosolic antioxidant capacity, and altered synthesis and mitochondrial phenotype are some potential cellular mechanisms of exercise-induced cardiac protection $[3,8]$. However, the exact mechanism for the positive effects of exercise is unclear and has remained a serious challenge.

Autophagy is the elimination of damaged organelles and proteins with lysosomes. The autophagy process requires a family of proteins called Autophagy-Related Genes (ATGs). ATG6 or Beclin-1 gene is the most important ATG in mammals for autophagy. Under the normal circumstances, the autophagy process facilitates the replacement of functionally impaired organelles with healthy organelles. However, in pathological conditions, excessive autophagy causes erratic cellular deletion and eventually cell death. Therefore, autophagy balance is important to maintain proper cell function [9].

Based on the previous studies, various cellular mechanisms affect IR injury; one of which is the autophagy process disruption in cardiac cells $[3,10]$. During IR, a high level of autophagy occurs which may increase cardiomyocyte injury after IR [10]. Therefore, preventing autophagy balance disruption during IR can reduce cellular and tissue damages. Exercise training can significantly affect cellular autophagy balance [11-13].

Studies report that exercise training reduces the myocardial infarct size in laboratory rats $[5,8]$, and provides neuroprotection in cerebral ischemic injury via autophagy suppression [13]. Exercise training has obvious benefits in maintaining the autophagy process balance [1113]. Exercise training, by modulating cellular autophagy, may play a cardiovascular protection role from IR injury. Thus, the current study evaluated the preconditioning effect of high-intensity aerobic training on myocardial IR injury and autophagy, as a potential exercise-induced cardiac protection mechanism.

\section{Materials and Methods}

\section{Study samples}

This was an experimental study with a control group. The samples were 32 eight-week-old male Wistar rats. 
Rats were kept under normal conditions (temperature: $22 \pm 2^{\circ} \mathrm{C} ; 12: 12 \mathrm{~h}$ light-dark cycle) with free access to food and water.

\section{Study procedure}

After one week of familiarity with the laboratory environment, rats were randomly divided into 4 groups of 8 , as follows: 1 . Training (Tr) group, where the rats were trained on a rodent treadmill for 8 weeks, then underwent IR surgery (Tr plus IR); 2. Control (Ctr) group with IR operation, where the samples received no training, then underwent IR operation (Ctr plus IR); 3. sham group, where the samples received no training, then underwent IR operation without coronary artery occlusion (the surgical conditions were simulated in this group); and 4. the control group, where rats received no training, then were anesthetized without IR surgery.

Tr plus IR group was anesthetized by the intraperitoneal injection of thiopental sodium $(50 \mathrm{mg} / \mathrm{kg})$ at 48 hours after the last training session. Different sets of rats from each group were used for morphological and biochemical analyses (half of them for morphological and the rest for biochemical test).

\section{Training protocol}

Training plus IR group received high-intensity aerobic training on a rodent treadmill at the speed of $34 \mathrm{~m} / \mathrm{min}$ $\left(80-85 \% \mathrm{VO}_{2 \max }\right)$ at $0^{\circ}$ incline for 8 weeks; five days a week and 60 min per session. Previous studies suggest that aerobic training should be performed at speeds greater than $60 \% \mathrm{VO}_{2 \max }$ to demonstrate cardiac protection properties [6]. Therefore, we used this protocol for high-intensity training. During the first week of training, the rats practiced at the speed of $10 \mathrm{~m} / \mathrm{min}$ for $15 \mathrm{~min}$ (adaptation period).

During the second and third weeks, the intensity and duration of the exercise gradually increased to reach the final speed and duration ( $34 \mathrm{~m} / \mathrm{min}, 60 \mathrm{~min}$ per day) at the end of week three (overload period). From week 8 onward, the speed and duration of training were kept constant (stabilization period). The rats were trained for $10.2 \mathrm{~km}$ per week [14].

\section{IR operation}

IR operation was conducted on the rats that had been selected for morphological tests. The rats were intubated after anesthesia with sodium thiopental and a small animal ventilator (Model 683, Harvard Apparatus, USA) was used for breathing. The volume was set at 5-6 milliliters and the respiratory rate was set at $60-70$ beats per minute.

A thermal pad was used to maintain the body temperature at $37^{\circ} \mathrm{C}$ during the operation. After stabilizing the respiratory state, an incision on the animal chest was made in the space between the third and fourth ribs to access the Left Anterior Descending (LAD) coronary artery. A silk suture (6.0) was passed around the LAD, and a transparent polyethylene catheter was used to form a snare. Then, ischemia was maintained for $30 \mathrm{~min}$. After releasing the snare, the heart was reperfused for $90 \mathrm{~min}$ [5].

The time periods used for ischemia and reperfusion were inspired by the previous studies that reported if the duration of ischemia is greater than $20 \mathrm{~min}$, the cardiac cells will be irreversibly affected by the processes of apoptosis and necrosis, and myocytes death will occur [3]. After reperfusion, the LAD was re-occluded, followed by the injection of $2 \mathrm{~mL}$ Evans blue dye (SigmaAldrich, St Louis, MO, USA) into the inferior vena cava to delineate the ischemic area as a tissue area without the blue dye. Eventually, the heart was removed and stored at $-20^{\circ} \mathrm{C}$ for one day until analysis.

\section{Infarct size measurement}

To measure the infarct size and risk areas, the frozen heart was cut into 5 or 6 transverse slices from apex to base (1-mm thick). Then, to stain viable cells in the risk zone, the slices were immersed in sodium phosphate buffer and both sides of each slice were photographed by a scanner. Afterward, the slices were incubated in triphenyltetrazolium chloride (TTC, Sigma-Aldrich, St Louis, $\mathrm{MO}, \mathrm{USA}$ ) at $37^{\circ} \mathrm{C}$ for $20 \mathrm{~min}$ to colorize the living cells in the at-risk area. After incubation, the slices were immersed in $10 \%$ formalin for one day, then were rescanned to show the infarct size (a part of the at-risk area that remains white after incubation with TTC solution).

The extent of at-risk areas and infarct size were calculated using ImageJ (for each slice, the average score of these indicators on both sides was considered). The risk area was expressed as a percentage of total ventricle area, while the infarct area was expressed as a percentage of the at-risk area $[5,15]$.

Gene expression measurement using the real-time polymerase chain reaction technique

To extract RNA from the heart tissue, Kiazol solution (Kiazist Co., Iran) was employed. According to RNA extraction instructions, in several sequential steps, 
Table 1. Characteristics of the primers used in the real-time polymerase chain reaction

\begin{tabular}{cccc}
\hline Gene & Primer Sequence & Product Size (bp) & Accession Number \\
\hline \multirow{2}{*}{ Beclin-1 } & $\begin{array}{c}\text { Forward TGGCACAGCGGACAATTGG } \\
\text { Reverse AACAGTACAACGGCAACTCCTTA }\end{array}$ & 232 & $>$ NM_001034117.1 \\
\multirow{2}{*}{ B-actin } & $\begin{array}{l}\text { Forward ATCAGCAAGCAGGAGTACGAT } \\
\text { Reverse AAAGGGTGTAAACGCAGCTC }\end{array}$ & 94 & NM_031144.3 \\
\hline
\end{tabular}

chloroform, isopropanol, and ethanol solutions (Merck Millipore, USA) were used in several centrifugation steps. After confirming the concentration and purity of the extracted RNA using Thermo Scientific NanoDrop One UV-Vis spectrophotometers, the cDNA synthesis kit (GeneAll, South Korea) was used to convert $1 \mu \mathrm{g}$ of RNA into cDNA.

In this study, the expression of beclin- 1 and $\beta$-actin genes were measured. Table 1 presents the primer sequences of these genes. Afterward, the efficiency of primers was evaluated by qPCR master mix (Ampliqon, Denmark) by applying the real-time polymerase chain reaction technique (LightCycler 96, Roche Life Sciences, Germany). Then, using this method, the amplification of genes were measured in triplicate.

At the end of the evaluation, the melting curve peaks of each gene were analyzed to identify specific products from non-specific ones. In all cases, the single-peak polymerase chain reaction product was observed, indicating that the reaction product was completely specific. CT index of beclin-1 was normalized as a reference gene, and the relative expression of beclin-1 was calculated based on the Pfaffl method [16].

\section{Data analysis}

Normal distribution of data was examined by the Shapiro-Wilk test. To examine the possible difference between groups, 1-way Analysis of Variance (ANOVA) and Bonferroni test were performed in SPSS. The significance level was set at $\mathrm{P}<0.05$ and data were presented as $\mathrm{Mean} \pm$ SD.

\section{Results}

Characteristics of the samples are listed in Table 2. There is no significant difference between the studied rats in terms of initial body weight, final body weight, and cardiac weight $(\mathrm{F}=0.6,2.1$, and $1.3 ; \mathrm{P}=0.66,0.09,0.27$, respectively). The ratio of cardiac weight to body weight $(\mathrm{mg} / \mathrm{g})$ was higher in the Tr plus IR group $(\mathrm{F}=4.4, \mathrm{P}=0.006)$.

According to Figure 1, risk areas were similar in all groups after aerobic training $(\mathrm{F}=0.03, \mathrm{P}=0.9)$. The obtained results (Figure 2) indicated that infarct size was larger in the Ctr+IR and Tr+IR groups, compared to the sham group $(\mathrm{F}=113.3, \mathrm{P}=0.001)$. However, it was smaller in the Tr plus IR group $(20.24 \pm 5.7 \%)$ compared to the Ctr plus IR group $(35.9 \pm 2.3 \%)(\mathrm{P}=0.001)$. Beclin-1 gene expression ratio (Figure 3) was reported higher in the Ctr plus IR group compared to other groups. However, its difference was not significant in the Tr plus IR group compared to the control and sham groups, and aerobic training could significantly reduce Beclin-1 gene expres$\operatorname{sion}(\mathrm{F}=81.2, \mathrm{P}=0.001)$.

\section{Discussion}

This study evaluated the effect of high-intensity aerobic training as a preconditioning factor on IR injury and au-

Table 2. The characteristics of the study rats categorized by groups

\begin{tabular}{|c|c|c|c|c|c|c|}
\hline \multirow{2}{*}{ Category } & \multicolumn{4}{|c|}{ Mean \pm SD } & \multirow{2}{*}{$\mathbf{F}$} & \multirow{2}{*}{ Sig. } \\
\hline & Control & Sham & Ctr Plus IR & Tr Plus IR & & \\
\hline Initial body weight, g & $212.2 \pm 9.3$ & $213.9 \pm 8.4$ & $214.9 \pm 10$ & $214.3 \pm 9.7$ & 0.6 & 0.66 \\
\hline Final body weight, $g$ & $335.6 \pm 26.7$ & $314.3 \pm 15.7$ & $338.2 \pm 36.3$ & $309.7 \pm 22.9$ & 2.1 & 0.09 \\
\hline Cardiac weight , g & $1.21 \pm 0.12$ & $1.07 \pm 0.10$ & $1.17 \pm 0.16$ & $1.24 \pm 0.16$ & 1.3 & 0.27 \\
\hline The ratio of cardiac weight to body weight, $\mathrm{mg} / \mathrm{g}$ & $3.61 \pm 0.2$ & $3.42 \pm 0.3$ & $3.48 \pm 0.4$ & $4 \pm 0.3^{*}$ & 4.4 & 0.006 \\
\hline
\end{tabular}

* Significant compared to the control, sham, and Ctr plus IR groups at $\mathrm{P}<0.05$ 


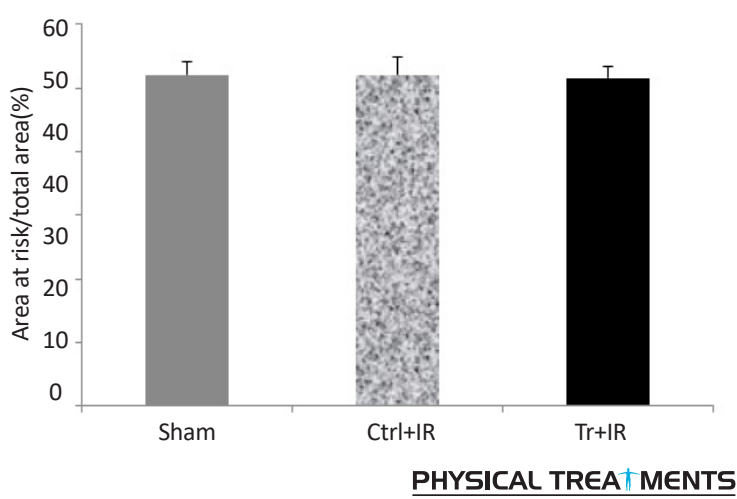

Figure 1. Myocardial area at risk vs. total myocardial area in the study groups $(\mathrm{Mean} \pm \mathrm{SD})$

tophagy as a possible mechanism for exercise-induced cardiac protection. The ratio of cardiac weight to body weight of the studied rats was measured to assess exercise-induced cardiac growth. This ratio was higher in the training group compared to other groups, which reveals the effectiveness of aerobic exercise training on cardiac muscles.

The IR operation significantly induced myocardial infarction. Moreover, eight-week aerobic training protected the heart from IR injury. The size of the myocardial risk area was the same among the different study groups. However, the infarct size is an appropriate indicator of cardiac resistance from IR injury. Our results on the effect of exercise-induced cardiac protection were consistent with previous studies, indicating that exercise can protect the heart from arrhythmia and infarction caused by $\operatorname{IR}[5,17-19]$.

Data analysis was performed at 48 hours after the last training session in the Tr plus IR group to eliminate the acute effects of exercise. Therefore, gene expression changes can be attributed to chronic adaptations. The

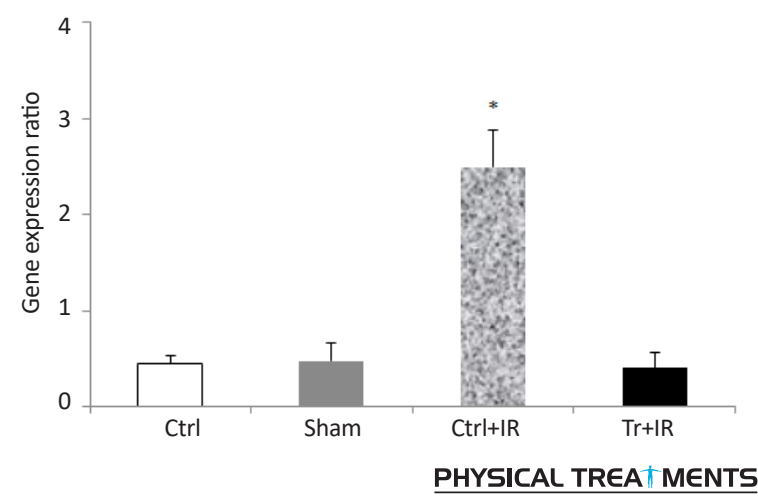

Figure 3. Beclin-1 gene expression ratio in the study groups Mean \pm SD

* Significant compared to the control, sham, and Tr plus IR groups $(\mathrm{P}<0.05)$

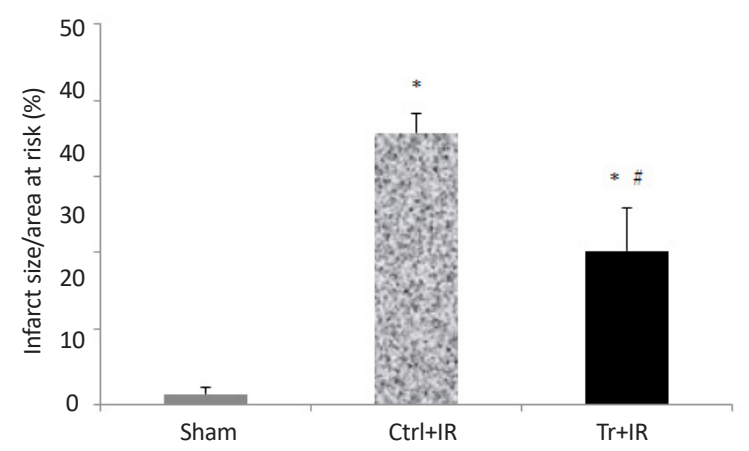

PHYSICAL TREA TMENTS

Figure 2. Infarct size vs. myocardial area at risk in the study groups (Mean \pm SD)

* Significant compared to the sham group $(\mathrm{P}<0.05)$; \# Significant compared to the Ctr plus IR group $(\mathrm{P}<0.05)$.

current study indicated that beclin-1 gene expression ratio, as the main indicator of the cellular autophagy process, was higher in the Ctr plus IR group compared to control and sham groups. However, in the Tr plus IR group, its expression was not different from that of the control and sham groups. This indicates that aerobic training can reduce its expression level after IR injury.

Our study was the first to assess the role of autophagy in exercise-induced cardiac protection against IR injury. Zhang et al. (2014) studied the role of exercise pretreatment in the autophagy process after cerebral ischemia. They concluded that a 2 -week exercise plan can provide neuroprotection in cerebral ischemic injury. They also studied autophagy signaling as a possible mechanism. Their results revealed that exercise, by reducing the autophagy process in the cell, could play a role in cardiac protection from IR injury [13].

Lu et al. (2018) argued that exercise preconditioning can reduce exhaustive exercise-induced myocardial injuries. They suggested that the down regulation of autophagy may reduce myocardial injury induced by exhaustive exercises [11]. Autophagy by the degradation of cytoplasmic material plays an important role in cell decomposition in case of the lack of energy which is essential to maintaining the tissue natural function. However, studies revealed that an excessive autophagy process is required when IR injury occurs, which increases the level of damage to cardiomyocyte injury after IR [10].

Some mechanisms have been proposed for exerciseinduced cardiac protection $[3,5,17]$. The autophagy imbalance can influence myocardial infarction development during IR injury [20, 21], preventing the disruption in the autophagy process. This can maintain the normal functioning of cardiac cells and reduce the injury caused 
by IR. It is unclear how the accelerated autophagy process increases IR-induced cardiac injuries. However, it has been demonstrated that the induction of autophagy is redox (oxidant and antioxidant balance) sensitive.

That is, the increased generation of reactive oxygen species in the cell leads to cellular proteolytic systems activation. Furthermore, exercise improves cell antioxidant capacity; thereby inhibiting or delaying autophagic system activation [22]. According to the obtained results, aerobic training can protect the heart from IR injury. It seems that autophagy balance improvement resulting from the aerobic training plays a role in this event. Maintaining the balance of the autophagy process by aerobic training can contribute to reducing IR-induced cardiac injury. In other words, it can protect the heart from IR injury by preventing autophagy process disruption. Further studies are required to address the different aspects of the autophagy process as a potential mechanism for exercise-induced cardiac protection.

\section{Ethical Considerations}

\section{Compliance with ethical guidelines}

All study interventions were implemented according to the National Health Association's guide for the care and use of laboratory animals. The laboratory protocol was also approved by the Ethics Committee of Hamadan University of Medical Sciences (Code: IR.UMSHA. REC.1396.549).

\section{Funding}

This research was financially supported by Hamadan University of Medical Sciences and University of Guilan.

\section{Authors contributions}

All authors contributed in preparing this article.

\section{Conflict of interest}

The authors declared no conflict of interest.

\section{Acknowledgements}

Authors would like to thank the personnel of the Neurophysiology Research Center of Hamadan University of Medical Sciences for their valuable cooperation.

\section{References}

[1] Dorweiler B, Pruefer D, Andrasi TB, Maksan SM, Schmiedt W, Neufang A, et al. Ischemia-Reperfusion injury. European Journal of Trauma and Emergency Surgery. 2007; 33(6):60012. [DOI:10.1007/s00068-007-7152-z] [PMID]

[2] Hatmi ZN, TahvildariS, Motlag AG, Kashani AS. Prevalence of coronary artery disease risk factors in Iran: A population based survey. BMC Cardiovascular Disorders. 2007; 7(1):32

[3] Powers SK, Smuder AJ, Kavazis AN, Quindry JC. Mechanisms of exercise-induced cardioprotection. Physiology. 2014; 29(1):27-38. [DOI:10.1152/physiol.00030.2013] [PMID] [PMCID]

[4] Lennon SL, Quindry JC, French JP, Kim S, Mehta JL, Powers SK. Exercise and myocardial tolerance to ischaemia-reperfusion. Acta Physiologica Scandinavica. 2004; 182(2):161-9. [DOI:10.1111/j.1365-201X.2004.01346.x] [PMID]

[5] Esposito F, Ronchi R, Milano G, Margonato V, Di Tullio $\mathrm{S}$, Marini M, et al. Myocardial tolerance to ischemia-reperfusion injury, training intensity and cessation. European Journal of Applied Physiology. 2011; 111(5):859-68. [DOI:10.1007/s00421-010-1707-0] [PMID]

[6] Kavazis AN. Exercise preconditioning of the myocardium. Sports Medicine. 2009;39(11):923-35. [DOI:10.2165/11317870000000000-00000] [PMID]

[7] Tao L, Bei Y, Lin S, Zhang H, Zhou Y, Jiang J, et al. Exercise training protects against acute myocardial infarction via improving myocardial energy metabolism and mitochondrial biogenesis. Cellular Physiology and Biochemistry. 2015; 37(1):162-75. [DOI:10.1159/000430342] [PMID]

[8] Ghahremani R, Damirchi A, Salehi I, Komaki A, Esposito F Mitochondrial dynamics as an underlying mechanism involved in aerobic exercise training-induced cardioprotection against Ischemia-Reperfusion injury. Life Sciences. 2018 213:102-8. [DOI:10.1016/j.lfs.2018.10.035] [PMID]

[9] Wu JJ, Quijano C, Chen E, Liu H, Cao L, Fergusson MM, et al. Mitochondrial dysfunction and oxidative stress mediate the physiological impairment induced by the disruption of autophagy. Aging. 2009; 1(4):425-37. [DOI:10.18632/agg ing.100038] [PMID] [PMCID]

[10] Gatica D, Chiong M, Lavandero S, Klionsky DJ. Molecular mechanisms of autophagy in the cardiovascular system. Circulation Research. 2015; 116(3):456-67. [DOI:10.1161/ CIRCRESAHA.114.303788] [PMID] [PMCID]

[11] Lu J, Pan SS, Wang QT, Yuan Y. Alterations of cardiac KATP channels and autophagy contribute in the late cardioprotective phase of exercise preconditioning. International Heart Journal. 2018; 59(5):1106-15. [DOI:10.1536/ihj.17-003] [PMID]

[12] Yan Z, Lira VA, Greene NP. Exercise training-induced regulation of mitochondrial quality. Exercise and Sport Sciences Reviews. 2012; 40(3):159-64. [DOI:10.1097/ JES.0b013e3182575599]

[13] Zhang L, Niu W, He Z, Zhang Q, Wu Y, Jiang C, et al Autophagy suppression by exercise pretreatment and p38 inhibition is neuroprotective in cerebral ischemia. Brain Research. 2014; 1587:127-32. [DOI:10.1016/j.brainn res.2014.08.067] [PMID] 
[14] Garekani ET, Mohebbi H, Kraemer RR, Fathi R. Exercise training intensity/ volume affects plasma and tissue adiponectin concentrations in the male rat. Peptides. 2011; 32(5):1008-12. [DOI:10.1016/j.peptides.2011.01.027] [PMID]

[15] Ranjbar K, Zarrinkalam E, Salehi I, Komaki A, Fayazi B. Cardioprotective effect of resistance training and Crataegus oxyacantha extract on ischemia reperfusion-induced oxidative stress in diabetic rats. Biomedicine \& Pharmacotherapy. 2018; 100:455-60. [DOI:10.1016/j.biopha.2018.02.021] [PMID]

[16] Pfaffl MW. A new mathematical model for relative quantification in real-time RT-PCR. Nucleic Acids Research. 2001; 29(9):e45. [PMID] [PMCID]

[17] Brown DA, Jew KN, Sparagna GC, Musch TI, Moore RL. Exercise training preserves coronary flow and reduces infarct size after Ischemia-Reperfusion in rat heart. Journal of Applied Physiology. 2003; 95(6):2510-8. [DOI:10.1152/japplt physiol.00487.2003] [PMID]

[18] Lee Y, Min K, Talbert EE, Kavazis AN, Smuder AJ, Willis WT, et al. Exercise protects cardiac mitochondria against Ischemia-Reperfusion injury. Medicine and Science in Sports and Exercise. 2012; 44(3):397-405. [DOI:10.1249/ MSS.0b013e318231c037] [PMID]

[19] Rahimi M, Shekarforoush S, Asgari AR, Khoshbaten A, Rajabi H, Bazgir B, et al. The effect of high intensity interval training on cardioprotection against Ischemia-Reperfusion injury in wistar rats. EXCLI Journal. 2015; 14:237-46. [DOI;10.17179/excli2014-587] [PMID] [PMCID]

[20] Wang JY, Xia Q, Chu KT, Pan J, Sun LN, Zeng B, et al Severe global cerebral ischemia-induced programmed necrosis of hippocampal CA1 neurons in rat is prevented by 3-methyladenine: A widely used inhibitor of autophagy. Journal of Neuropathology \& Experimental Neurology. 2011; 70(4):314-22. [DOI:10.1097/NEN.0b013e31821352bd] [PMID]

[21] Wen YD, Sheng R, Zhang LS, Han R, Zhang X, Zhang XD, et al. Neuronal injury in rat model of permanent focal cerebral ischemia is associated with activation of autophagic and lysosomal pathways. Autophagy. 2008; 4(6):762-9. [DOI:10.4161/auto.6412] [PMID]

[22] Smuder AJ, Kavazis AN, Min K, Powers SK. Doxorubicininduced markers of myocardial autophagic signaling in sedentary and exercise trained animals. Journal of Applied Physiology. 2013; 115(2):176-85. [DOI:10.1152/japplphysii ol.00924.2012] [PMID] 